
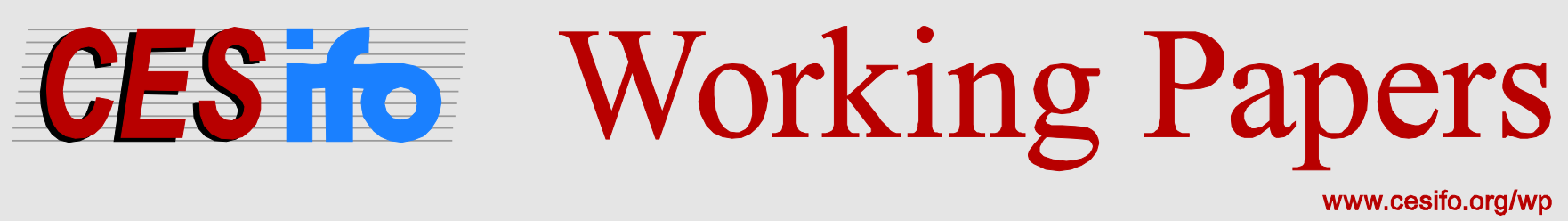

\title{
Pricing Internet Traffic: Exclusion, Signalling and Screening
}

\author{
Bruno Jullien \\ Wilfried Sand-Zantman
}

CESIFO WORKING PAPER NO. 4709

CATEGORY 11: INDUSTRIAL ORGANISATION

MARCH 2014

An electronic version of the paper may be downloaded

- from the SSRN website:

- from the RePEc website:

- from the CESifo website:

wWw.SSRN.com

www.RePEc.org

www.CESifo-group.org/wp

\section{CESifo}




\title{
Pricing Internet Traffic: Exclusion, Signalling and Screening
}

\begin{abstract}
We consider a network that intermediates traffic between free content providers and consumers. While consumers do not know the traffic cost when deciding on consumption, a content provider knows his cost but may not control the consumption. We study how pricing consumers' and content providers' sides allows both profit extraction from the network and efficient information transmission. In the case of uniform tariff, we argue that a positive pricecap on the charge to content is optimal (with no constrain on the consumer side). Proposing menus helps signaling useful information to consumers and therefore adjusting consumption to traffic cost. In the case of menus, we show that optimal mechanisms consist in letting the content producers choose between different categories associated with different prices for content and consumers. Our results are robust to competition between ISPs and to competition between contents. We also show that when (competitive) content providers choose at small cost between a pay and a free business model, a price-cap at cost on the price for content improves efficiency.
\end{abstract}

JEL-Code: D000.

Bruno Jullien

Toulouse School of Economics

(CNRS-GREMAQ \& IDEI)

31000 Toulouse / France

bruno.jullien@tse-fr.eu
Wilfried Sand-Zantman

Toulouse School of Economics

(GREMAQ \& IDEI)

31000 Toulouse / France

wsandz@tse-fr.eu

June 2012, revised February 2014

This paper had circulated before under the title "Congestion Pricing and Net Neutrality". We thank Byung-Cheol Kim, Jan Krämer, Martin Peitz, Mike Riordan, David Salant and Tommaso Valletti for helpful discussions and comments, as well as participants at 2012 CRESSE Conference, CREST-LEI seminar, The Future of Internet Conference (MaCCI, Mannheim), ICT 2012 Conference (ParisTech), EARIE 2013 Conference and the 2nd London IO Day. We gratefully acknowledge Orange, in particular Marc Lebourges, for its intellectual and financial support under the IDEI/Orange convention. 


\section{Introduction}

The pricing of traffic on Internet is the object of intense debate, with contrasted views on the way the operators of the physical network should treat various contents and on the relationship between content providers and Internet service providers. The economic debate stems from the difficulty to reconcile the need for traffic management due to increasing volume with the original open and neutral nature of Internet. This paper provides a new perspective on the issue by focusing on the informative role of prices charged to consumers and content providers.

Large successes on Internet have involved business strategies based on services offered for free to consumers and financed by third-party advertising (obvious examples are Google, Yahoo News or Facebook). When consumers don't pay for the content, there is no price to signal scarcity to consumers and value to producers. To this extent, we may view Internet as an instance of markets with some missing prices, implying some misallocation of resources.

Efficient traffic management must ensure that the various actors on the web internalize the cost they impose on the ecosystem. This is not straightforward in a network, as the cost of communications results from the interaction between two agents, i.e. the consumer who receives the traffic and the content provider who sends the traffic. The way consumption is transformed into costs depends on factors that are usually known and controlled by the content provider. Hence consumers can hardly forecast the cost they impose at the time they choose consumption. ${ }^{1}$ A standard procedure for commercial services of this nature would be to charge the cost to the seller, inducing a pass-through into consumer price. However, with no price charged by the content provider and thus no pass-through, alternative solutions to signal costs must be considered.

A first alternative scenario would be for the ISP to measure the load at the level of each content provider and than inform the consumers before they make their consumption choice. This seemingly natural solution turns to be difficult in practice. Indeed, one of the principles of internet is the end-to-end principle. This has two direct consequences. First, while the network may know that the consumer is on a video website, it cannot know the precise video downloaded and the bandwidth required. Second, if the network were using deep packet inspection and monitoring services, he could not inform consumers at the request level.

In this paper, we study how the use of tariffs concerning both consumers and content providers can play this signalling role and indeed provide useful information for consumers to promote efficient network use.

\footnotetext{
${ }^{1}$ There is a distinction between what a consumer perceives as content and what a network perceives as a cost. The consumer may care about a video, a voice message or some news article, while the network views bits of information.
} 
We consider a network that intermediates the traffic between content providers and consumers. The content is heterogenous in the cost of traffic (referred to as the load). Consumers know their preferences but not the load generated by their consumption; a content provider knows his load but has no direct control on the consumption. Content providers may receive income proportional to traffic, such as advertising revenue or direct utility for the producer, but do not charge a retail price for content.

The network observes only the cost of traffic but not the consumption nor the load. Based on the observed cost of traffic the network can charge a price to one or both of the parties involved in traffic generation. We assume that the network can charge a hook-up fee to consumers and consider in turn the case of monopoly and then of competitive content providers.

We first discuss the use of a simple pair of tariff, one part being paid by consumers and the other one by content providers. ${ }^{2}$ This pair is chosen so that first efficient consumption prevails and second most of the content providers' profit is extracted. We show that this leads to excluding some content providers and discuss the impact of several price regulations on this context. In particular a zero price for content is suboptimal compared to a price-cap regulation imposed on the traffic charge imposed to content.

Then, we characterize a mechanism designed to achieve transmission of the right signal to consumers. It involves screening different types of contents by a menu of tariffs and making the tariffs transparent to consumers, referred to as "category pricing". A practical example of such an optional tariff is the AT\&T's Sponsored Data program recently announced by ATT that allows content providers to pay for the data used by their customers, that would then not be counted in their subscriber's monthly data limits. We show that an optimal allocation can be implemented by the network through offering a menu of categories associated with pairs of prices for receiver and sender. Each content provider chooses a category, and consumers are informed of the category prior to consumption. The price paid by consumers decreases with the price paid by the content. Faced to the menu, each content provider must trade-off the volume of consumption with the cost of traffic. The lowest load content will then opt for the highest consumption while the highest load content will opt for the lowest cost.

Our analysis applies to both monopoly and competing ISPs, to both non-rival and competitive content providers. We also allow the content providers to choose between paid and free services, and show that when this choice entails little cost, a price-cap at cost on the traffic price for content providers improves the outcome.

\footnotetext{
${ }^{2}$ In most countries, fixed and mobile network operators offer consumers a menu of tariffs with different traffic allowance, thus non-linear tariffs. We abstract from this issue by considering only two-part tariffs.
} 
Related literature Our work is related to the two-sided market literature (see Armstrong (2006), Caillaud and Jullien (2003), Rochet and Tirole (2003, 2006)), as we try to understand how both sides of the market, the content providers and the consumers, must be priced. Our model is mixed between the participation model of Armstrong (2006) and the usage model of Rochet and Tirole (2003). Indeed, in our basic model, the total number of agents on the consumer's side is fixed and only the consumption is affected by the price and the number (more precisely the types) of content providers on the market. On the content providers' side, the profit depends on the price the platform charges but also on the number of consumers and the price charged to them by the network. And the number of content providers can vary, as too high a price may exclude the high load contents.

In the field of telecommunication, some previous work has been developed to study the pricing structure between receivers and senders. This literature has emphasized the importance of call externalities, and therefore the social benefit of using positive receiver prices (see Jeon, Laffont and Tirole (2004)). Hermalin and Katz (2004) develop a related idea but focusing on the way to deal with the uncertainty of the private value of exchanging messages and the gaming, i.e. the choice to call or to wait for a call, induced by the tariffs structure. In our paper, the structure of communication is different as it is the receiver (the consumer) who is at the origin of the communication. Moreover the sender is the only one to know the cost of this communication. The presence of both moral hazard and adverse selection makes our setting quite different from the previous articles studying the pricing of communication.

The literature on the pricing on Internet has been driven by the debate over net neutrality and the optimal way to price content providers and consumers (see Economides and Hermalin (2012)). One point that emerges from two-sided market models is that while laissez-faire will not result in efficient pricing, the precise nature of the intervention that would foster efficiency is unclear (see Economides and Tag (2012) for instance). Neglecting the investment question (on this point, see Choi and Kim (2010) or Hermalin and Katz (2009), and the literature below), we focus on the efficient management of current resources when there is an uncertainty on the real cost of consumption. By focusing on the information revelation aspect of prices, we offer a new perspective that complements previous studies on the impact of price discrimination (see Hermalin and Katz (2007)). Several recent contributions discuss the screening of traffic sensitive contents by means of prices and differentiated quality layers, a key aspect of the net-neutrality debate (see Bourreau, Kourando and Valletti (2014), Kämer and Wiewiorra (2010), Choi, Jeon and Kim (2013), Reggiani and Valletti (2012), Peitz and Schuett (2013)). Our contribution departs from these by considering consumption usage and the informational role of consumer prices. A specific contribution is to show that screening among traffic sensitive contents can be achieved with different consumer prices and a single 
quality layer.

One particular feature of our analysis is the uncertainty on the total load, which results both from the load parameter of the contents and of the consumption behavior of consumers. There is thus a flavor of screening in a common value environment (see Akerlof (1970), Rothshild and Stiglitz (1976) or Samuelson (1984) for the classical cases). As the trade is intermediated by the platform, our paper is related to the study of auctions with a monopoly broker and correlated value between sellers and buyers as studied in Jullien and Mariotti (2006). The common value element is not intrinsic to our problem but is endogenously created by the pricing system by which the cost of each sides is a function of both the load parameter and the consumption behavior.

The next section presents the model. Section 3 discusses the role of pricing both consumers and content providers when the network uses a simple linear and uniform rule to price traffic. Section 4 develops the idea of category pricing and shows how the category should be optimally designed by the network and what would be the impact of regulating internet pricing. Section 5 proposes three extensions, first by considering elastic subscription demand and competition at the ISP level, then by studying the impact of competition at the content level and finally by endogenizing the content providers' choice of business model (pay vs free). Section 6 concludes.

\section{Model}

\subsection{Presentation}

We analyze the tariff charged for traffic by a network (in the case of Internet, an Internet Service Provider (ISP)) to two sides of the market: consumers and content providers. In practice, some contents are delivered freely while others are paid for, an issue that we will discuss in the last section. As this is the main originality and the focus of this paper, we simplify the analysis by assuming that all contents are free. For the main part of the paper, we also assume that contents are non rival so that consumers visit every contents. The expected demand for each content when consumers face a price $p$ per unit of content is $E(q)=D(p)$. We assume that $D($.$) is decreasing and convex { }^{3}$, the consumption $D(0)$ of free goods is positive finite, and demand vanishes at price $\bar{p}>0$. The expected consumer surplus for a given content at price $p$ is then given by $S(p) \equiv \int_{p}^{\bar{p}} D(s) d s$.

\footnotetext{
${ }^{3}$ The convexity assumption is mainly made to simplify the analysis but it is not necessary for most of the results and our main conclusions can be extended to the case of non-convex demand.
} 
We assume that each unit of consumption generates a net benefit $a>0$ for the content provider. This benefit includes the advertising revenue ${ }^{4}$ and other benefits of the content provider but also the cost of distributing the content if any. Any transaction between a content provider and a consumer generates a cost for the network. More precisely for any unit of consumption, each content will generate an expected cost $\theta$, referred to as the load, to the network: the consumption of $q$ units of content with a load $\theta$ generates a cost $\theta q$ to the network. ${ }^{5}$ The cost may be direct or related to congestion. One view is that the network needs to expand resources to maintain the quality of service and that $\theta$ reflects this need. ${ }^{6}$ In a set-up with explicit congestion, $\theta$ would be interpreted as the shadow cost of congestion.

We assume that content providers can be of two types, $\ell$ and $h$ which are characterized by the two components, $\theta$ and $a$. A content is type $\ell$, i.e. $\left(\theta_{\ell}, a_{\ell}\right)$, with probability $1-\lambda$ while the content is type $h$, i.e. $\left(\theta_{h}, a_{h}\right)$, with probability $\lambda$. The type of each content is unknown to consumers and the network, but known to the content provider. We assume that the two types of content providers can be ordered using the ratio between advertisement revenue and load, denoted $b$, with

$$
b_{\ell}=\frac{a_{\ell}}{\theta_{\ell}} \leq b_{h}=\frac{a_{h}}{\theta_{h}}
$$

In the sequel, we will use $\left(\theta_{i}, b_{i}\right)$ as parameters characterizing the content of type $i$. We will refer to contents of type $h$ as high benefits (in short HB) contents while contents of type $\ell$ will be referred to as low benefits (in short LB) contents. As this is the most interesting case, we also assume that one type of content could not be proposed if the providers had to pay the full cost of traffic:

$$
b_{\ell}<1<b_{h}
$$

This assumption captures the idea that some content providers may afford paying the cost and not others. Thus it is not possible that all contents are proposed unless part of the cost of traffic is paid by the consumers.

While the content provider has information about the load, the level of consumption is determined by consumers. Moreover, the network cannot monitor $\theta$ and $q$ but observes only the ex-post realization of $\operatorname{cost} \theta q$ and can charge any side for this cost. We restrict attention

\footnotetext{
${ }^{4}$ Advertising revenue increases with consumption if the time spend on the page by consumers increases with consumption, or if advertising is tied with consumption.

${ }^{5}$ For example, $q$ may be the number of songs downloaded by the consumer while $\theta$ is the bandwidth taken by each song. Alternatively one may view $q$ as a number of subscriptions and $\theta$ the traffic generated by one subscription.

${ }^{6}$ When the cost is only related to congestion, one may view $\theta q$ as a cost that the network will bear ex-post to maintain the traffic.
} 
to linear traffic prices, that is prices of the form $s \theta q$ to the content providers and $r \theta q$ to the consumers, where the unit prices $s$ and $r$ are non-negative. We denote by $\Pi$ the variable profit of the network, defined as the difference between of the traffic revenues and costs.

We assume that the network (the ISP) is able to charge an ex-ante hook-up fee $F$ to consumers for subscription.

In most of our analysis, we will consider the timing below

1. The network proposes the prices $r$ and $s$, and the hook-up fee $F$.

2. Consumers decide to affiliate or not.

3. Each content provider observes his type, i.e. his $\theta$ and $a$, and decides to be active or not.

4. Consumers observe the choice of each content provider and choose how much to consume of each content.

5. Traffic is observed, payments to the network take place.

The extent to which the hook-up fee allows the network to capture an increase in consumer surplus that traffic management generates depends on various factors, in particular the elasticity of participation to the network and the competition at the ISP level. In the main part of this analysis, we focus on the case of inelastic participation and discuss more general setting latter. We will show in section 5 that the choice of variable tariffs $s$ and $r$ depends neither on the assumption of inelastic participation on the consumers' side nor on the absence or presence of competition at the ISP level.

Let us denote by $C S$ the consumer expected surplus from usage (gross of the hook-up fee). We assume for the moment that consumers are ex-ante identical and risk neutral, so that the consumers' subscription decision is based solely on $C S-F$. In this case the network can extract the full expected surplus with the hook-up fee $F=C S$. It is then optimal for the network to maximize the joint expected surplus with consumers and to use the hook-up fee to share this surplus with the consumers.

As a consequence the network's objective fully internalizes the surplus of consumers. We therefore ignore the fee $F$ and assume for now on that the network maximizes the sum of its variable profit $\Pi$ and consumer expected surplus $C S$, denote $V=C S+\Pi$ and refer to it as the network value. ${ }^{7}$ As we will develop latter, the behavior of the ISP will lead to the

\footnotetext{
${ }^{7}$ We use the term network value because, although it coincides with the network total profit in our basic model, this will not be the case with elastic demand and with competition.
} 
maximization of $V$ even under competition between ISPs, whenever consumers do not have private information about their expected surplus before joining the network.

At last, we assume that entry is always efficient if priced at the true marginal cost for any possible positive value of $a$, i.e., $\max \left\{\theta_{\ell}, \theta_{h}\right\}<\bar{p}$.

\subsection{Socially optimal prices}

As a benchmark, we consider the socially optimal prices in the case of a single content with full information on the content type and no direct transfer between content providers and consumers. This would correspond to the situation of a regulated network maximizing social welfare. For a content with type $(\theta, a=b \theta)$, the price perceived by consumers is given by $r \theta$. The content generates a gain $a-s \theta=(b-s) \theta$ per unit of consumption and thus is proposed only if $s \leq b$. Social welfare then writes as

$$
S(r \theta)+(r \theta-\theta+b \theta) D(r \theta) \text { s.t. } s \leq b=\frac{a}{\theta} .
$$

Ignoring feasibility constraints, this leads to

$$
r=1-b \text { and } s \leq b
$$

As consumers face a unit price $1-b$, this tariff induces efficient consumption. The difficulty with the above solution is that it may involve negative prices when the benefits are high.

When negative prices cannot be used, we obtain directly:

Lemma 1 Under full information, the socially (constrained) optimal allocation is obtained by charging $r=\max \{1-b, 0\}$ and $s \leq b$.

Notice that setting $s=b$ generates an allocation that is (constrained) efficient and such that the content providers receive zero surplus. The value $V=C S+\Pi$ is then equal to the maximal welfare $W$. This implies that the network maximizing $V$ implements the social optimum under full information about $\theta$. We now investigate the case of imperfect information. 


\section{Uniform pricing}

\subsection{Equilibrium prices and exclusion}

When the content provider does not charge for its product, it generates its profit only through the benefits. Absent any price for traffic, all content providers are active, but a positive price $s$ may induce some exclusion. Notice that as the content providers do not charge any price to consumers, the price $s$ charged by the network to content providers is not reflected in an equivalent increase in the cost supported by consumers. This has two implications:

1. If the network wishes to reduce the consumption, it has to do so with a price $r>0$ to consumers; the reduction is then uniform across contents.

2. If the network wishes to reduce selectively the consumption of LB contents, it can only do so with a price $s$ high enough that the LB content providers exit from the market.

In this section, we are restricting ourselves to a single price $r$ and a single price $s$ that are determined ex-ante. Facing a price $s$, a content provider of type $i$ stays on the market if it anticipates a non-negative profit, hence if $s \leq b_{i}$ for $i=\ell, h$. This implies that the volume of free content is:

$$
M(s)= \begin{cases}1 & \text { if } s \leq b_{\ell} \\ \lambda & \text { if } b_{\ell}<s \leq b_{h}\end{cases}
$$

and the average data load is given by

$$
\mathbb{E}(\theta \mid s)= \begin{cases}\theta^{e}(\lambda)=(1-\lambda) \theta_{\ell}+\lambda \theta_{h} & \text { if } s \leq b_{\ell} \\ \theta_{h} & \text { if } b_{\ell}<s \leq b_{h}\end{cases}
$$

In what follows we omit the argument $\lambda$ unless this is needed for clarity. Increasing the tariff $s$ above $b_{\ell}$ excludes the LB contents and thus reduces the average load. ${ }^{8}$

In a rational expectation equilibrium, consumers correctly anticipate the mean load and the data cost $r \mathbb{E}(\theta \mid s)$. As they do not pay for content, they will consume $D(r \mathbb{E}(\theta \mid s))$ for each content. We can then write the consumer surplus and the profit as

$$
C S=M(s) \times S(r \mathbb{E}(\theta \mid s)), \Pi=M(s) \times(r+s-1) \mathbb{E}(\theta \mid s) D(r \mathbb{E}(\theta \mid s)) .
$$

\footnotetext{
${ }^{8}$ Notice here that, as there is no fixed cost of entry, the market structure on the content producers side (monopoly or competition) does not affect the analysis. All results of this section, derived in the case where each CP is a monopoly, extend therefore fully to the case of competitive CPs.
} 
The network maximizes the joint surplus with consumers

$$
V=M(s) \times[S(r \mathbb{E}(\theta \mid s))+(r+s-1) \mathbb{E}(\theta \mid s) D(r \mathbb{E}(\theta \mid s))]
$$

The term $S(r \mathbb{E}(\theta \mid s))+(r+s-1) \mathbb{E}(\theta \mid s) D(r \mathbb{E}(\theta \mid s))$ captures the incentives to maximize the per content joint surplus of the network and consumers for a given value of $s$. The expected data cost per content is $(1-s) \mathbb{E}(\theta \mid s)$ and internal efficiency is achieved by setting a consumer price equal to this cost whenever feasible. As content participation is independent of the consumer price, the network chooses:

$$
r=\max \{1-s, 0\}
$$

When $s \leq 1$, we have $V=M(s) . S((1-s) \mathbb{E}(\theta \mid s))$ which is the consumer surplus accounting for the cost reduction that the tax on content generates. The network chooses $s$ by comparing two prices for content, the maximal price $s=b_{\ell}$ that maintains full participation with $r=1-b_{\ell}$, and the maximal price $s=b_{h}>1$ that preserves participation of the HB contents only with $r=0$. The respective network value are:

$$
\begin{aligned}
V_{\lambda}\left(b_{\ell}\right)= & S\left(\left(1-b_{\ell}\right) \theta^{e}\right) \text { if } s=b_{\ell} \\
& \text { and } \\
V_{\lambda}\left(b_{h}\right)= & \lambda\left[S(0)+\left(b_{h}-1\right) \theta_{h} D(0)\right] \text { if } s=b_{h}
\end{aligned}
$$

Note that with the low price $\left(s=b_{\ell}\right)$, there is no exclusion but some rent is left to the HB contents and consumers bear a high share of data cost. On the other hand, with the high price $\left(s=b_{h}\right)$, the LB contents are excluded but all the HB contents' rent is captured by the network and consumers support a low share of data cost. Intuitively, the gain from excluding the LB content should increase with proportion of $\mathrm{HB}$ contents and indeed we find:

Proposition 1 There exists $\lambda^{*}$ strictly between 0 and 1, such that the network excludes the $L B$ contents (with a price $s=b_{h}$ ) if $\lambda>\lambda^{*}$.

Proof. Denoting $R=r \mathbb{E}(\theta \mid s)$ the expected cost for consumers, we have

$$
V_{\lambda}\left(b_{\ell}\right)=S\left(\left(1-b_{\ell}\right) \theta^{e}\right)
$$


From the envelop theorem to differentiate, we find

$$
\frac{\partial V_{\lambda}\left(b_{\ell}\right)}{\partial \lambda}=\left(b_{\ell}-1\right) D\left(\left(1-b_{\ell}\right) \theta^{e}\right)\left(\theta_{h}-\theta_{\ell}\right)
$$

and

$$
\frac{\partial^{2} V_{\lambda}\left(b_{\ell}\right)}{\partial \lambda^{2}}=\left(b_{\ell}-1\right) \max \left\{1-b_{\ell}, 0\right\} D^{\prime}\left(\left(1-b_{\ell}\right) \theta^{e} \theta^{e}\right)\left(\theta_{h}-\theta_{\ell}\right)^{2} \geq 0 .
$$

which has slope 0 if $b_{\ell} \geq 1$ and $-\left(b_{\ell}-1\right)^{2} D^{\prime}\left(\left(1-b_{\ell}\right) \theta^{e}\right)\left(\theta_{h}-\theta_{\ell}\right)^{2}>0$ if $b_{\ell}<1$ is nondecreasing with $\lambda$. Thus $V_{\lambda}\left(b_{\ell}\right)$ is convex in $\lambda$, while $V_{\lambda}\left(b_{h}\right)$ is linear in $\lambda$.

Notice that $V_{0}\left(b_{\ell}\right)>V_{0}\left(b_{h}\right)=0$ (because demand is positive at price $\left(1-b_{\ell}\right) \theta_{\ell}$ and $V_{1}\left(b_{\ell}\right)<V_{1}\left(b_{h}\right)$ (because $\left.b_{\ell}<b_{h}\right)$. This implies that $V_{1}\left(b_{\ell}\right)<V_{1}\left(b_{h}\right)$ for $\lambda$ above a threshold $\lambda^{*}$

Typically the network will tax the content when the effect of exclusion on the average cost is large and when the average consumer surplus per unit of consumption is small.

\subsection{Welfare and regulation of content access}

\section{Regulated price for content under symmetric information}

The choice of the network is not optimal as it doesn't account for the surplus of the content. Indeed, using the definitions of $b_{h}$ and $b_{\ell}$, total welfare can be written as

$$
\begin{aligned}
W_{\lambda}\left(b_{\ell}\right) & =V_{\lambda}\left(b_{\ell}\right)+\lambda\left(b_{h}-b_{\ell}\right) \theta_{h} D\left(\left(1-b_{\ell}\right) \theta^{e}\right) ; \\
W_{\lambda}\left(b_{h}\right) & =V_{\lambda}\left(b_{h}\right) .
\end{aligned}
$$

Thus in our model, the network can appropriate the total welfare when the LB content is excluded. It then chooses consumer prices that maximize total welfare. On the other hand, when no content is excluded, some rent must be left to the HB contents corresponding to the difference between the benefit $a_{h}$ and the price paid $\theta_{h} b_{\ell}$. As an immediate consequence we find that:

Lemma 2 Under laissez-faire, there is excessive exclusion of content $\left(\lambda^{*}\right.$ is too low) and conditional on full participation, the consumer tariff is too large.

Proof. The first result follows from the fact that $W\left(b_{\ell}\right)>V_{\lambda}\left(b_{\ell}\right)$ while $W\left(b_{h}\right)=V_{\lambda}\left(b_{h}\right)$, implying that there is a range of parameter values for which the platform chooses to exclude while it would be efficient to choose $s=b_{\ell}$. The second follows from the fact than the socially optimal consumer price when there is no exclusion is $\theta^{e}-(1-\lambda) b_{\ell} \theta_{\ell}-\lambda b_{h} \theta_{h}<\theta^{e}-b_{\ell} \theta^{e}$. 
The network internalizes the consumer surplus and acts as a monopoly on behalf of the consumers. The revenue $s$ is then redistributed to consumers in the most efficient way, that is by a reduction of $r$ below 1, along with a fixed transfer that allows the network to capture the efficiency gain. As there is no price between the content providers and the consumers, the situation is akin to a two-sided market where the network extracts revenue on both sides. This network

i) wants to capture rents from the content providers which induces excessive exclusion,

ii) doesn't internalize all the benefits the content providers' derive from consumption so that $r$ is too high.

As exclusion can be excessive, it is natural to see whether some form of regulation can be useful. We shall discuss mostly in this paper the regulation of the price $s$ charged to content, leaving the consumer price unregulated. The second effect above implies that, conditional on the market structure, the socially optimal level of $s$ is one that induces the lowest consumer price $r$. As the consumer price decreases with the content price, this means that the maximal price $s$ compatible with the market structure is socially optimal when the consumer price is positive. Given that the unregulated content price is maximal at $b_{\ell}$ under full participation, regulation of $s$ cannot improve welfare when the network chooses this market structure, i.e. when $\lambda \leq \lambda^{*}$. Neither can it improve welfare when exclusion is socially optimal. However regulation may avoid exclusion of content by the network when this is suboptimal.

Let us now derive the socially optimal level of $s$.

Proposition 2 If a regulator controls $s$ only, then there exists $\lambda^{w}$ strictly between $\lambda^{*}$ and 1 such that exclusion is optimal for $\lambda>\lambda^{w}$. The optimal level of $s$ is either $b_{\ell}$ with no exclusion or any $s \in\left[1, b_{h}\right]$ with exclusion.

Proof. See appendix

Thus, whenever full participation is not compatible to full data cost recovery on the content side $\left(b_{\ell}<1\right)$, an exclusionary price for content may be efficient. The reason for that is that the consumer price is too high when there is no exclusion. To see that, notice that when $s=b_{\ell}$, the consumer price $r=1-b_{\ell}$ is strictly positive and the demand for content is smaller than $D(0)$ which would be optimal for the HB content. Setting $s \geq 1$ has the inconvenient of excluding the LB contents but it raises efficiency for the HB contents by reducing the consumer price to zero and restoring optimal demand $D(0)$. When the mass 
$\lambda$ of HB contents is large, the latter effect dominates and exclusion raises welfare as well as the value appropriated by the network. ${ }^{9}$

In practice regulators may be uncertain about some parameters so that they may not know precisely whether $\lambda$ is above $\lambda^{w}$, or the precise values of $b_{\ell}$ or $b_{h}$. So we consider here simple forms of regulation.

\section{Price-cap under asymmetric information on $\lambda$}

Proposition 2 shows that there always exists an optimal level of $s$ less than or equal to one. This suggests that some form of limitation of the price is optimal. In this part we investigate the role of price-cap. To address this issue, we assume that the regulator is uncertain about the proportion $\lambda$ of HB contents and discuss whether some price-cap improves welfare uniformly over $\lambda$.

For a given price-cap $\sigma \in\left(b_{\ell}, b_{h}\right)$ on the content price $s$, the network compares $V_{\lambda}\left(b_{\ell}\right)$ with $V_{\lambda}(\sigma)$. As in the unregulated case we have:

Lemma 3 For $\sigma \in\left(b_{\ell}, b_{h}\right)$ there exists $\lambda^{c}(\sigma) \in\left(\lambda^{*}, 1\right)$ such that under price-cap $\sigma$ on the content price, the network chooses to exclude the $L B$ content (by setting $s=\sigma$ ) if $\lambda>\lambda^{c}(\sigma)$. The threshold $\lambda^{c}(\sigma)$ is decreasing in $\sigma$ on $\left(b_{\ell}, b_{h}\right)$ from 1 to $\lambda^{*}$.

Proof. The proof is the same as for Proposition 1 replacing $V_{\lambda}\left(b_{h}\right)$ by

$$
V_{\lambda}(\sigma)=\max _{r \geq 0}\left[S\left(r \theta_{h}\right)+(r+\sigma-1) \theta_{h} D\left(r \theta_{h}\right)\right]
$$

Then we have

$$
S\left(\left(1-b_{\ell}\right) \theta^{e}\left(\lambda^{c}(\sigma)\right)\right)=\lambda^{c}(\sigma) \max _{r \geq 0}\left[S\left(r \theta_{h}\right)+(r+\sigma-1) \theta_{h} D\left(r \theta_{h}\right)\right]
$$

implying that $\lambda^{c}(\sigma)$ decreases with $\sigma$ because the RHS increases with $\sigma$. By definition $\lambda^{*}=$ $\lambda^{c}\left(b_{h}\right)$. Moreover

$$
S\left(\left(1-b_{\ell}\right) \theta^{e}\left(\lambda\left(b_{\ell}\right)\right)\right)=\lambda^{c}\left(b_{\ell}\right) \max _{R \geq 0}\left[S(R)+\left(R+b_{\ell}-1\right) \theta_{h} D(R)\right]=\lambda^{c}\left(b_{\ell}\right) S\left(\left(1-b_{\ell}\right) \theta_{h}\right)
$$

which holds at $\lambda^{c}\left(b_{\ell}\right)=1$ and $\theta^{e}=\theta_{h}$.

Thus tightening the price-cap allows reducing the range of exclusion, which we have shown to be too large. Optimal exclusion arises when $\lambda^{c}(\sigma)=\lambda^{w}$. The difficulty is to achieve the

\footnotetext{
${ }^{9}$ In the paper we assume that $b_{\ell}<1$. If on the contrary $b_{\ell}>1$, the network chooses $s>1$ and $r=0$. It is then immediate that $s \in\left[1, b_{\ell}\right]$ with no exclusion is socially optimal.
} 
optimal level of exclusion while inducing the socially optimal level of consumption. Indeed the same instrument $\sigma$ must control the choice to exclude and the price $r=(1-\sigma, 0)$.

Notice then that when $\lambda^{c}(\sigma)=\lambda^{w}$ at a price-cap $\sigma \geq 1$, reducing exclusion is compatible with maintaining optimal consumption, and thus $r=0$, under exclusion. This is the case whenever $\lambda^{c}(1) \geq \lambda^{w}$. Even when this is not the case, one can always improve on laissez-faire:

Lemma 4 A price-cap $\sigma \geq 1$ such that $\lambda^{c}(\sigma) \leq \lambda^{w}$ weakly dominates laissez-faire for all $\lambda$.

Proof. This follows from the fact the consumer price is $r=0$ under exclusion and $\lambda^{*}<$ $\lambda^{c}(\sigma) \leq \lambda^{w}$.

Thus it is always possible to impose a price-cap above the cost of traffic that improves welfare. Whenever $\lambda^{c}(1) \geq \lambda^{w}$, the optimal price allocation of Proposition 2 can be implemented with the correct choice of price-cap. Choosing a price-cap when $\lambda^{c}(1)<\lambda^{w}$ is more complex as reducing $\sigma$ below 1 creates an inefficiency. The trade-off is then between reducing the scope for exclusion (raising $\lambda^{c}(\sigma)$ ) and avoiding an increase in $r$ above zero for $\lambda>\lambda^{c}(\sigma)$. To illustrate this, suppose that $\lambda^{c}(1)<\lambda^{w}$ and the regulator evaluates that $\lambda$ is distributed according to a density $f(\lambda)$.

Proposition 3 An optimal price-cap is such that i) $\sigma>1$ with $\lambda^{c}(\sigma)=\lambda^{w}$ if $\lambda^{c}(1) \geq \lambda^{w}$, ii) $\sigma=1$ if $\lambda^{c}(1)<\lambda^{w}$ and the regulator's objective puts enough weight on $\lambda \in\left[\lambda^{c}(1), 1\right]$ and iii) $1 \geq \sigma>b_{\ell}$ otherwise.

Proof. See appendix.

Notice that a fixed price regulation at $s=1$ (and thus $r=0$ ) is clearly dominated by a price-cap $\sigma=1$, because it would induce exclusion of contents with a benefit $b_{\ell}<1$, despite a positive social value.

\section{Net-neutrality}

The result shades also light on some aspect of the net-neutrality debate. One view that has been expressed is that only consumers should pay for traffic. We thus define such a net-neutrality regulation as a zero-price rule for content ( $s=0$ in our setting). As it is a particular form of price-cap, it is immediate that in our context, a positive price-cap dominates the net-neutrality regulation. A price-cap may however be difficult to implement if the regulator has little information. In this case, a question is whether zero-price regulation dominates laissez-faire.

When $s=0$, the network sets a consumer price $r=1$. Using $a^{e}=(1-\lambda) b_{\ell} \theta_{\ell}+\lambda_{h} b_{h} \theta$, surplus is then

$$
W_{\lambda}(0)=S\left(\theta^{e}\right)+a^{e} D\left(\theta^{e}\right) .
$$


The optimality of a zero price over exclusion depends on the sign of $W_{\lambda}(0)-W_{\lambda}\left(b_{h}\right)$, which is convex in $\lambda$, positive when $\lambda=0$ and negative at $\lambda=1$ (as $W_{\lambda}\left(b_{h}\right)$ is the maximal welfare when $\lambda=1$ ). Hence the regulation with $s=0$ dominates exclusion of the LB contents if $\lambda$ is below a threshold $\lambda_{0}$.

Notice that as $W_{\lambda}(0)<W_{\lambda}\left(b_{\ell}\right)$, if $\lambda_{0}<\lambda^{*}$, there will be no value of $\lambda$ for which the policy may be optimal. We then have

Proposition 4 Laissez-faire (weakly) dominates net-neutrality $(s=0)$ if $\lambda^{*} \geq \lambda_{0}$ or if $\lambda \notin\left(\lambda^{*}, \lambda_{0}\right)$. Moreover $\lambda^{*} \geq \lambda_{0}$ if $b_{\ell}$ and $b_{h}$ are close, while $\lambda^{*}<\lambda_{0}$ if $b_{\ell}$ is small.

Proof. See appendix.

When the benefits $b_{i}$ of both types of content are quite similar, the price chosen by the ISP when he decides to exclude the LB content is close to the price that induces full participation. The ISP will then only exclude the LB contents when the mass of HB contents is very large, i.e. $\lambda^{*}$ is high. For those high values of $\lambda$, it is socially optimal to exclude the $\mathrm{LB}$ contents so there is no reason to impose any regulation.

Consider now the case where the low benefit is small. The ISP is then able to extract a much higher amount from a HB content than from a LB content. This makes exclusion of the $\mathrm{LB}$ content more attractive for the network, i.e. $\lambda^{*}$ is low and regulation can be welfare enhancing. ${ }^{10}$

\section{Cost-orientation}

One common form of regulation is based on the principle that the revenue from access should not exceed the cost. In the context of our model, we need to account for the two sources of revenue, consumers and content. We then define:

Definition 1 (Two-sided cost-orientation) Under cost-orientation the total revenue from traffic cannot exceed the total cost.

Cost-orientation is thus viewed here as a global price-cap on the two sides of the market. As prices cannot be negative, under cost-orientation, the network must set $s \leq 1$. Notice that when this is the case, the profit maximizing consumers price is $r=1-s \geq 0$. It follows that cost-orientation is formally equivalent to a price-cap $\sigma=1 \mathrm{imposed}$ on the content price with no restriction on the consumer side. Therefore it is dominated by a price-cap on $s$. Still it is interesting to see when it would improve on laissez-faire.

\footnotetext{
${ }^{10}$ We also show in appendix that the comparison is ambiguous when $b_{h}$ is large because in this case both the ISP and the regulator may exclude the LB content, the former to extract more rent from the HB content, the latter to raise consumption of $\mathrm{HB}$ content by lowering the price charged to consumers.
} 
From the previous analysis of price-cap, under cost-orientation the network chooses to exclude the LB content (by setting $s=1$ ) if $\lambda>\lambda^{c}(1)$, where $\lambda^{c}(1)>\lambda^{*}$ is defined in Lemma 3. It is quite immediate that cost-orientation is neutral when $\lambda<\lambda^{*}$. The reason is that it binds only when $s \geq 1$ is the optimal price. Cost orientation has a real effect only when it changes the choice of the ISP from exclusion to no exclusion which requires $\lambda^{*}<\lambda<\lambda^{c}(1)$. We then find:

Proposition 5 Cost-orientation weakly dominates laissez-faire if $b_{h}$ is small enough. However, if $b_{h}$ is large enough, $\lambda^{c}(1)>\lambda^{w}$ and cost-orientation reduces welfare for $\lambda \in\left(\lambda^{w}, \lambda^{c}(1)\right)$.

Proof. See appendix.

Capping the price $s$ is either neutral or improves welfare for all $\lambda<\lambda^{w}$. However it may prevent efficient exclusion for $\lambda^{w}<\lambda<\lambda^{c}(1)$, when demand is very elastic and the benefits that the HB content providers derive from consumption is large. In this case, as discussed in proposition 3 , a price-cap on $s$ at $\sigma>1$ may dominate cost-orientation.

\section{Discrimination with Category pricing}

With uniform pricing, the network were facing a trade-off between capturing the rent of HB content providers (with high $s$ and low $r$ ) and avoiding the exclusion of the LB content providers (with low $s$ and high $r$ ). One way to alleviate this trade-off, and to transmit precise information to consumers, is to allow the network to propose more complex tariffs. To this end, we consider the possibility for the network to achieve second-degree discrimination between the different types of content providers by offering a menu of linear tariffs. Obviously, there is no possibility to discriminate between different content providers without inducing some differential consumptions. Indeed, if consumers are not affected by the choices of the content providers, the consumption would be the same for all contents and all content providers would always opt for the smallest price $s$. However, the network may try to raise its profits and the value offered to consumers by combining a higher price for the contents with a lower price for consumers. Content providers eager to generate traffic (due in our model to a low $\theta$ or high benefits $b$ ) may then opt for this option. The advantage for the network may be not only to extract more rent from content providers but also to induce more efficient levels of consumption. We define this strategy as "category pricing".

Category pricing: The network proposes two tariffs $\left(s_{\ell}, r_{\ell}\right)$ and $\left(s_{h}, r_{h}\right)$. The content provider chooses a tariff, the consumer observes the tariff and consumes. 
Category pricing then amounts to define several classes or categories, $\ell$ and $h$. Content providers choose which category they want to belong to and this information is transmitted to the consumers. The tariffs then depend on the category.

Notice that if the network succeeds in inducing the LB and the HB content providers to choose different categories, then consumers should eventually realize that the average load is different for the two categories. They will thus adapt their behavior to the price of the category but also to the load in the category. This interaction between screening on one side and signalling on the other side is the difference between category pricing and a standard screening model.

As in the above section we focus on the non-rival content case, i.e. assuming that for each type of content, there is a unique provider, whose characteristics are privately known by the content provider. In this setting, we define a separating category tariff $\left\{\left(s_{\ell}, r_{\ell}\right),\left(s_{h}, r_{h}\right)\right\}$ as one for which there exists an allocation of prices and consumption levels with the two properties below:

1. Consumers anticipate that the load is $\theta_{\ell}$ for the category $\ell$ and $\theta_{h}$ for the category $h$, and choose consumption accordingly for each category;

2. The LB (resp. HB) content providers choose category $\ell$ (resp. $h$ ), given consumption in each category;

3. Consumptions are positive for both categories.

The first condition imposes that the consumers perfectly anticipate the traffic load by observing the category chosen by the content provider (hence a rational expectation equilibrium). This implies that we have consumptions $q_{\ell}=D\left(r_{\ell} \theta_{\ell}\right)$ and $q_{h}=D\left(r_{h} \theta_{h}\right)$ in the categories $\ell$ and $h$ respectively.

To highlight the difference with a uniform exclusionary tariff (that also achieves separation), we further impose that both types of content are active in a separating category tariff:

$$
0<q_{\ell}<q_{h} .
$$

Defining $U$ as the representative consumer utility function ${ }^{11}$, the network value is then:

$$
\begin{aligned}
& \lambda\left[U\left(D\left(r_{h} \theta_{h}\right)\right)+\left(s_{h} \theta_{h}-\theta_{h}\right) D\left(r_{h} \theta_{h}\right)\right]+(1-\lambda)\left[U\left(D\left(r_{\ell} \theta_{\ell}\right)\right)+\left(s_{\ell} \theta_{\ell}-\theta_{\ell}\right) D\left(r_{\ell} \theta_{\ell}\right)\right] \\
& { }^{11} U(D(p))=S(p)+p D(p) .
\end{aligned}
$$


For what follows, it will be convenient to think of a tariff in terms of prices for content $\left(s_{\ell}, s_{h}\right)$ and consumption levels $\left(q_{\ell}, q_{h}\right)$.

Then we have a separating tariff if the tariffs induce participation

$$
b_{\ell} \geq s_{\ell} \text { and } b_{h} \geq s_{h}
$$

and the following incentive compatibility conditions hold: ${ }^{12}$

$$
\begin{aligned}
\left(b_{\ell}-s_{\ell}\right) \theta_{\ell} q_{\ell} & \geq\left(b_{\ell}-s_{h}\right) \theta_{\ell} q_{h} \\
\left(b_{h}-s_{h}\right) \theta_{h} q_{h} & \geq\left(b_{h}-s_{\ell}\right) \theta_{h} q_{\ell}
\end{aligned}
$$

A tariff inducing an allocation that violates the condition (2) is equivalent to uniform prices. Indeed uniform prices with full participation of contents can be represented with prices for content $s_{\ell}=s_{h} \leq b_{\ell}$ and consumptions $q_{\ell}=q_{h}=D\left(r \theta^{e}\right)$. Second any uniform prices with exclusion of the LB content may be represented with prices $s_{\ell}=b_{\ell}, s_{h} \geq b_{\ell}$ and consumption $q_{\ell}=0$ and $q_{h}=D\left(r_{h} \theta_{h}\right)$. In both cases, the constraints (3) and (4) are satisfied but not (2). It follows that we may represent all options available to the network with prices for content $\left(s_{\ell}, s_{h}\right)$ and consumption levels $\left(q_{\ell}, q_{h}\right)$ that satisfy $(3)$ and (4).

When screening, the network maximizes the value under the participation constraints and the incentive compatibility constraints.

Then the program of the network becomes

$$
\begin{aligned}
& V=\max _{s_{\ell}, s_{h}, q_{h}, q_{\ell}} \lambda\left[U\left(q_{h}\right)+s_{h} \theta_{h} q_{h}-\theta_{h} q_{h}\right]+(1-\lambda)\left[U\left(q_{\ell}\right)+s_{\ell} \theta_{\ell} q_{\ell}-\theta_{\ell} q_{\ell}\right] \\
& \text { s.t. } \quad q_{h} \geq q_{\ell} \geq 0,(3) \text { and }(4) .
\end{aligned}
$$

The first constraint writes as $\left(b_{\ell}-b_{h}\right)\left(q_{\ell}-q_{h}\right)+\left(b_{h}-s_{\ell}\right) q_{\ell} \geq\left(b_{h}-s_{h}\right) q_{h}$, which implies (due to the second constraint) that $q_{\ell} \leq q_{h}$. From above, the program captures fully the options available to the network. Then a solution with $0<q_{\ell}<q_{h}$ corresponds to a separating category tariff, while other solutions correspond to uniform prices.

The program departs from the classical textbook cases as the transfers depend on the quantity and on the load $\theta$. Nevertheless, one can follow the usual procedure, with two possible cases, separating and pooling. Let us first ignore the constraint $q_{\ell} \leq q_{h}$. The

\footnotetext{
${ }^{12}$ Notice that a revealing allocation may induce $s_{\ell}=s_{h}$ as long as $r_{\ell}=r_{h}=0$. In this case the CPs are indifferent between revealing their types to consumers or not. Thus they can choose category $\ell$ or $h$ depending on their type although there is no payoff difference.
} 
solution then involves $s_{\ell}=b_{\ell},\left(b_{h}-s_{h}\right) q_{h}=\left(b_{h}-s_{\ell}\right) q_{\ell}$. Using these properties, we define the reduced program as:

$$
V=\max _{q_{h} \geq 0, q_{\ell} \geq 0} \lambda\left[U\left(q_{h}\right)+b_{h} \theta_{h} q_{h}-\left(b_{h}-b_{\ell}\right) \theta_{h} q_{\ell}-\theta_{h} q_{h}\right]+(1-\lambda)\left[U\left(q_{\ell}\right)+b_{\ell} \theta_{\ell} q_{\ell}-\theta_{\ell} q_{\ell}\right] .
$$

(Program R)

This directly leads to the proposition below (using $\left.U^{\prime}\left(q_{i}\right)=r_{i} \theta_{i}\right)$.

Lemma 5 The solution of the reduced program $R$ is such that:

$$
\begin{aligned}
& s_{\ell}=b_{\ell}, r_{\ell}=1-b_{\ell}+\frac{\lambda}{1-\lambda} \frac{b_{h}-b_{\ell}}{\theta_{\ell}} \theta_{h} \\
& s_{h}=b_{h}\left(1-\frac{q_{\ell}}{q_{h}} \frac{\theta_{\ell}-\theta_{h}}{\theta_{\ell}}\right), r_{h}=0 .
\end{aligned}
$$

The menu proposed by the ISP plays two roles. It allows a screening of the different types of content providers and at the same time it sends the right signal to consumers on the cost their consumption generates. Consider first the tariff designed for the HB content providers. As the benefits generated by the provider is higher than the cost, the network wants to induce consumption and therefore sets zero prices for receivers. As far as the price paid by the content providers is concerned, the price $s_{h}$ is smaller than $b_{h}$ because the platform must leave some profit to the $\mathrm{HB}$ content providers to induce truthful revelation. The price $s_{\ell}$ paid by the LB content providers is set so as to minimize the profit left while the price $r_{\ell}$ paid by consumers to access these contents has two origins. First, it reflects, as in the case of uniform tariff, the net cost of any unit of consumption. But this price is also distorted to minimize the level of profit one has to leave to the HB content providers and still induce truthful revelation. As it is common in the information economics literature, informational asymmetries lead the network to propose higher prices and therefore generate social costs.

Note that at the solution of the reduced program, the network may decide to exclude the LB content providers. This happens when the quantity $q_{\ell}$ resulting from the price characterized in lemma 5 is below 0 , and thus when $\lambda$ is large:

$$
q_{\ell}=0 \Leftrightarrow \lambda>\bar{\lambda}=\frac{\bar{p}-\left(1-b_{\ell}\right) \theta_{\ell}}{\bar{p}-\left(1-b_{\ell}\right) \theta_{\ell}+\left(b_{h}-b_{\ell}\right) \theta_{h}} .
$$

The reduced program thus characterizes all separation solutions, with exclusion or with category pricing. This solution is valid as long as the quantities are increasing, i.e. $q_{\ell} \leq q_{h}$. Note however that $q_{h}=D(0)$ which is the maximum quantity so the quantity cannot be decreasing with types and the incentive compatibility conditions are satisfied for all the types of contents with the separating menu. It follows that the solution of the reduced program is 
the optimal contract. ${ }^{13}$

The main question is whether it is indeed optimal for the network to use category pricing. The alternative is exclusion of the LB content with a tariff $s=s_{h}$.

Proposition 6 If category pricing is allowed, the network excludes the LB contents for $\lambda>\bar{\lambda}>\lambda^{*}$, otherwise it opts for a separating category tariff with efficient consumption of $H B$ contents and downward distortion of the consumption of the LB contents. Exclusion is all the more likely that $\theta_{\ell}$ are high, or that $b_{\ell}$, or $\theta_{h}$ are low.

Proof. See appendix

We now turn to the welfare analysis of category pricing, in particular compared to the uniform pricing policy. Note that $\bar{\lambda}>\lambda^{*}$ means that the ISP will exclude less often than with uniform prices.

Therefore, from a welfare perspective,n we find that:

Proposition 7 Comparing category pricing with the case of a uniform tariff,

- if $\lambda>\bar{\lambda}$ : then welfare is unchanged;

- if $\bar{\lambda}>\lambda>\lambda^{*}$ : category pricing increases welfare, the rent of the HB contents is larger and the LB contents stay in the market.

- if $0<\lambda<\lambda^{*}$, category pricing increases welfare if $\lambda$ is small enough.

Proof. See appendix

First note that when there is exclusion ( $\lambda$ high), allowing category pricing does not improve welfare.

As it is now standard in the analysis of price-discrimination, category pricing raises welfare if it avoids the exclusion of the LB content. The new feature is that the HB contents also benefit from the discrimination. The reason here is that only second-degree discrimination is allowed. Thus when allowing the LB contents to stay with a low $s$ (when $\bar{\lambda}>\lambda>\lambda^{*}$ ) the network needs to leave some rent to the HB content providers that were not needed with exclusionary uniform prices.

When there is no exclusion, the effect of screening is more ambiguous. On one hand consumers are better informed about the cost of traffic which raises efficiency by allowing to reduce the price for the $\mathrm{HB}$ contents. However the consumption of LB contents is reduced which may or may not raise welfare. Notice that when $\lambda$ is small, the distortion of $q_{\ell}$ (or equivalently of $r_{\ell}$ ) is small and the former effect dominates, making category pricing the optimal system.

\footnotetext{
${ }^{13}$ This would not be the case if $b_{h}<1$ in which case the monotonicity constraint could be binding.
} 


\section{Extensions}

\subsection{Elastic participation and competition between ISPs}

In the main analysis, we considered the case of a monopoly ISP with inelastic subscription demand. We want to show that introducing demand elasticity does not change the way variable cost is allocated between consumers and content providers, and thus the main conclusions of our work. The same holds for competition at the ISP level. To do so, we will give a more detailed description of the participation decision of the consumers.

More precisely, we consider a model with an initial mass 1 of consumers, a mass 1 of content providers (indexed by $x$ ) and $I \geq 1$ ISPs (indexed by $i$ ). The content providers are divided into a mass $\lambda$ of type $h$ and a mass $1-\lambda$ of type $\ell$. The utility of each consumer subscribing to ISP $i$ and consuming a consumption profile $\left\{q_{x}\right\}_{x}$ is given by

$$
\int_{x} u\left(q_{x}\right) d x+\varepsilon_{i}-F_{i}-T_{i} r_{i}
$$

$F_{i}$ represents the hook-up fee, $\varepsilon_{i}$ is an idiosyncratic shock, $T_{i}=\int_{x} \theta_{x} q_{x} d x$ the load and $r_{i}$ the variable price on this load. The idiosyncratic shock $\varepsilon_{i}$ is a random variable that represents the consumers' heterogeneity relative to the intrinsic taste for ISP $i$. We do not put any restriction on the distribution of the preference shocks, except that we implicitly assume that they do not convey any information about the utility from consuming contents.

The timing of the game is unchanged and in case there is competition between ISPS $(I>1)$, we assume that at stage 1 , each ISP $i$ chooses simultaneously an offer $\left(F_{i}, r_{i}, s_{i}\right)$. With this slightly modified setting, we assume that the content providers multi-home, paying only a variable price, while the consumers single-home. For conciseness we restrict to a single linear tariff but the argument below applies as well to category pricing.

If $N_{i}$ is the mass of consumers subscribing to ISP $i$, the profit of each content provider at ISP $i$ is given by

$$
N_{i}\left(a_{x}-s_{i} \theta_{x}\right) q_{x}=N_{i} \theta_{x}\left(b_{x}-s_{i}\right) q_{x}
$$

The content provider $x$ will choose to participate to ISP $i$ if $b_{x} \geq s_{i}$. It follows that as before the mass of content providers is $M\left(s_{i}\right)$. Moreover the expected load at ISP $i$ is $\mathbb{E}\left(\theta \mid s_{i}\right)=\theta^{e}$ if $s_{i} \leq b_{\ell}$ and $\mathbb{E}\left(\theta \mid s_{i}\right)=\theta_{h}$ if $b_{\ell}<s_{i} \leq b_{h}$.

With uniform tariff, the consumption of each content is the same, equal to $D\left(r_{i} \mathbb{E}\left(\theta \mid s_{i}\right)\right)$ and the surplus is given by

$$
C S_{i}=M\left(s_{i}\right) \cdot S\left(r_{i} \mathbb{E}\left(\theta \mid s_{i}\right)\right)
$$

A given consumer joining network $i$ gains $C S_{i}-\varepsilon_{i}-F_{i}$. As there are many potential 
networks,

$$
N_{i}=\operatorname{Pr}\left[C S_{i}-F_{i}+\varepsilon_{i} \geq \max \left\{0, \max _{j \neq i} C S_{j}-F_{j}+\varepsilon_{j}\right\}\right] .
$$

The total profit of ISP $i$ is given by

$$
N_{i} \cdot\left[F_{i}+M\left(s_{i}\right)\left(r_{i}+s_{i}-\mathbb{E}(\theta \mid s)\right) D\left(r_{i} \mathbb{E}\left(\theta \mid s_{i}\right)\right)\right]
$$

For any given strategy of the other ISP's - denoted $z_{-i}$ - let us define

$$
\phi_{i}\left(R ; z_{-i}\right)=\operatorname{Pr}\left[R \geq \max \left\{0, \max _{j \neq i} C S_{j}-F_{j}+\varepsilon_{j}\right\}-\varepsilon_{i}\right] .
$$

Then we can write the profit of ISP $i$ as

$$
\phi_{i}\left(C S_{i}-F_{i} ; z_{-i}\right) \cdot\left(F_{i}+M\left(s_{i}\right)\left(r_{i}+s_{i}-\mathbb{E}(\theta \mid s)\right) D\left(r_{i} \mathbb{E}(\theta \mid s)\right)\right) .
$$

With this formulation it easy to see that the ISP's best pricing strategy always maximizes the network value per consumer:

Proposition 8 In any equilibrium of the game with elastic subscription demand and I ISPs, each ISP chooses a tariff $\left(s_{i}, r_{i}\right)$ that maximizes the network value per consumer: $V_{i}=$ $M\left(s_{i}\right) \cdot\left\{S\left(r_{i} \mathbb{E}(\theta \mid s)\right)+\left(r_{i}+s_{i}-\mathbb{E}(\theta \mid s)\right) D\left(r_{i} \mathbb{E}(\theta \mid s)\right)\right\}$.

Proof. The profit can be written as

$$
\phi_{i}\left(R_{i} ; z_{-i}\right) \cdot\left[V_{i}-R_{i}\right]
$$

where $R_{i}=C S_{i}-F_{i}$ is the expected net consumer surplus and $V_{i}$ is the network value. Notice that $V_{i}$ is independent of the subscription fee $F_{i}$ and of other networks strategies $z_{-i}$, while $R_{i}$ depends on $F_{i}$. This implies that the network will always choose $\left(s_{i}, r_{i}\right)$ to maximize $V_{i}$.

The value $V_{i}$ only depends on the usage prices $\left(r_{i}, s_{i}\right)$ so there is a natural hierarchy in the pricing strategy. First the network maximizes the value that can be shared with consumers by setting adequate usage prices. Then the network decides how much surplus to retain and how much surplus to leave to the consumers. While the surplus $R_{i}$ left to consumers, and thus the subscription fee $F_{i}$, depends on the elasticity of demand and on the way competition is modeled between the ISPs, the prices $\left(r_{i}, s_{i}\right)$ do not. So the prices we derive in the main model assuming a monopoly ISP are also the equilibrium prices when they are more than one ISP competing for consumers. 
As far as welfare in concerned, for fixed total demand (inelastic consumers participation), introducing competition at the ISP level does not alter our results. But of course it may raise total participation to the market. Notice that, compared to the inelastic demand case, the analysis of the regulation of the traffic price should be more favorable to laissez-faire under competition.

Corollary 1 The regulation of data traffic tariffs should be more lenient when the aggregate demand is elastic.

The reason is that with elastic aggregate demand, increasing the value $V_{i}$ also increases subscription demand, an effect that we ignored in the above analysis. Thus the optimal price-cap, if any, will be higher and the case for allowing category pricing would be stronger.

\subsection{The case of competitive content providers}

We now investigate the situation where competition prevails for each type of content. We assume that, for each type of content, there is a large number $N$ of providers, all being characterized by the same type $\ell$ or $h$. The existence of competition at the content providers level has no impact as long as the tariffs proposed by the content providers are uniform so we focus on the case of category pricing.

A separating allocation is defined as in the case of monopoly content providers and the tariffs proposed by the network are still denoted $\left(s_{\ell}, r_{\ell}\right)$ and $\left(s_{h}, r_{h}\right)$ for each type. For those menus to be accepted, the participation constraints (3) should be satisfied. But, in contrast with the monopoly case, it is necessary to discuss the consumers' belief when one content provider chooses a different tariff than other providers of the same content. Suppose that the content is $\mathrm{HB}$ and all but one of its providers choose the HB contract. As it is common knowledge that all firms have the same cost, consumers will choose the firm proposing the lowest price $r{ }^{14}$ The same reasoning also applies to the LB content providers.

Let us first show that the ISP can propose the contract inducing full extraction of the content providers' profit, i.e. $\left(s_{h}=b_{h}, r_{h}=0\right)$ and $\left(s_{\ell}=b_{\ell}, r_{\ell}=1-b_{\ell}\right)$. Suppose HB content providers choose the HB tariff and consider first the behavior of any provider of this type. If it chooses the LB tariff, as $r_{\ell}>r_{h},{ }^{15}$ for any belief consumers could hold, the price they will pay by choosing this particular content provider will be greater than the one they would obtain by choosing any other. Therefore, the deviating HB firm will have no demand.

\footnotetext{
${ }^{14}$ This effect is connected to the standard Maskin effect in game where agents have private but correlated information, see Maskin (1999).

${ }^{15}$ To be precise, if $b_{h}>b_{\ell}>1$, then both $r_{h}=r_{\ell}=0$. To ensure the incentive compatibility condition, the ISP should set $r_{h}=\varepsilon$, with $r_{h}=\varepsilon$ as small as possible. Therefore in this case, the optimal contract converges to the first-best contract.
} 
Consider now the other incentive constraint concerning the LB content providers. As $s_{h}=b_{h}>b_{\ell}$, any deviation with positive sales on the HB tariff will entail a loss and thus is not profitable.

This shows that the exists a tariff and an equilibrium allocation such that the ISP extracts all the surplus from content providers and induces revelation of the type to consumers.

However this first-best contract does not implement the separating allocation as a unique equilibrium. Indeed, consider the equilibrium where all the content providers (both LB and HB) choose the contract designed for the LB content providers. The LB content providers would not gain by choosing the HB contract as they would make a loss on every transaction. The HB content providers make positive profit (given by $\left(b_{h}-b_{\ell}\right) \theta_{h}$ per consumer) by all choosing the LB contract while deviating to the HB tariff would generate zero profit. Thus, if we insist on unique implementation of the equilibrium allocation, full surplus extraction is not possible. However, for a large number of providers per content, it can be approximated.

Proposition 9 In the competitive case, the optimal tariff is given by $\left(s_{h}=b_{h}, r_{h}=0\right)$ and $\left(s_{\ell}=b_{\ell}, r_{\ell}=1-b_{\ell}\right)$. With a finite number $N$ of providers per content, unique implementation of the separating equilibrium is obtained by a tariff that converges to the previous tariff when $N$ goes to infinity.

Proof. Suppose the ISP proposes $\left(s_{\ell}=b_{\ell}, r_{\ell}=1-b_{\ell}\right)$ and $s_{h}>1$ with $r_{h}=0$. As $s_{h}>b_{\ell}$, the LB content providers choose the LB tariff. Then it is an equilibrium for the HB content providers to choose the LB tariff if for some beliefs $\theta$

$$
\frac{1}{N}\left(b_{h}-b_{\ell}\right) \theta_{h} D\left(r_{\ell} \theta^{e}\right) \leq\left(b_{h}-s_{h}\right) \theta_{h} D(0)
$$

This holds in particular if

$$
s_{h}=b_{h}-\frac{1}{N}\left(b_{h}-b_{\ell}\right) \frac{D\left(r_{\ell} \theta^{e}\right)}{D(0)}
$$

which converges to $b_{h}$ when $N$ goes to infinity.

Clearly, category pricing in this case yields a higher social welfare than any uniform tariff.

\subsection{Paid vs free content}

In what precedes we postulate that content providers face prohibitive costs for charging a price for their content (referred to as retail price). We now discuss the case where content providers may charge a retail price but with some transaction cost. We assume that each 
unit of payment generates a transaction cost $\mu \geq 0 .{ }^{16}$ As the object of the paper is not to discuss double marginalization issues and the exercise of market power at the content level, we maintain the assumption of competitive supply of content of Section 5.2. Thus there is a mass $\lambda$ of competitive $\mathrm{HB}$ contents and a mass $1-\lambda$ of competitive LB contents. We then denote by $p_{h}$ the competitive retail price for the $\mathrm{HB}$ content and $p_{\ell}$ the competitive retail price for the LB content, where the price applies to units of content (as opposed to traffic).

Notice that as long as $b_{\ell} \geq s$, all contents are offered for free because competition drives retail prices to zero as long as the net benefit per sale $b_{i}-s$ is positive. But if the traffic price is above $b_{i}$ then the type $i$ content provider will not exit but charge a positive retail price. Following this logic, for a given traffic price $s$, the content providers of type $i$ charge a retail price

$$
p_{i}=\max \left\{0, \theta_{i}\left(s-b_{i}\right)\right\}(1+\mu) .
$$

This implies that for any price $s$ above $b_{\ell}$, the competitive price will reflect the type of the content, i.e. consumers face retail prices $p_{\ell} \neq p_{h}$. When deciding how much to consume facing $r>0$, they must form some expectation over the traffic they will generate. For this, they may rely on the price of the content. If $p_{\ell} \neq p_{h}$, the price of the content reveals some information about traffic. A rational expectation equilibrium is then defined as an allocation such that:

$$
\left\{\begin{array}{l}
q_{i}=D\left(r \theta_{i}+p_{i}\right) \text { if } p_{\ell} \neq p_{h} \\
q_{i}=D\left(r \theta^{e}+p_{i}\right) \text { if } p_{\ell}=p_{h}
\end{array}\right.
$$

Equations (6) capture the idea that consumers will eventually realize that they tend to have more traffic when they consume more expensive content.

Consider now the optimal behavior of the network. If it chooses $s \leq b_{\ell}$, all contents are free and no information is transmitted to consumers so that the value is $V_{\lambda}(s)$, the same as in the case of free content. However any price strictly between $b_{\ell}$ and $b_{h}$ induces $p_{\ell}=\left(s-b_{\ell}\right) \theta_{\ell}(1+\mu)>p_{h}=0$. The price conveys information and the value is

$$
V=(1-\lambda)\left[S\left(r \theta_{\ell}+p_{\ell}\right)+(r+s-1) \theta_{\ell} D\left(r \theta_{\ell}+p_{\ell}\right)\right]+\lambda\left[S\left(r \theta_{h}\right)+(r+s-1) \theta_{h} D\left(r \theta_{h}\right)\right]
$$

Consider first the consumer traffic price The marginal value when $r$ changes is

$$
\frac{\partial V}{\partial r}=(1-\lambda)\left[\theta_{\ell}^{2} D^{\prime}\left(\left(r+s-b_{\ell}\right) \theta_{\ell}\right)+\lambda \theta_{h}^{2} D^{\prime}\left(r \theta_{h}\right)\right](r+s-1)
$$

implying that the network chooses $r=\max \{0,1-s\}$, as in the case of free content. Based

\footnotetext{
${ }^{16}$ This pertains only to consumers' payments. We maintain the assumption of zero transaction costs between the network and the content provider.
} 
on that we obtain for the choice of tariff $s$ :

Proposition 10 When the content may be paid, the network chooses between $\left(s=b_{\ell}, r=1-b_{\ell}\right)$ and a tariff $s^{p}(\lambda) \in\left[1, b_{h}\right]$ with $r=0$. The function $s^{p}(\lambda)$ is non-decreasing, with $s^{p}(0)=1$ and $s^{p}(1)=b_{h}$. For any $\lambda$, the network chooses $s^{p}(\lambda)$ if $\lambda \geq \lambda^{*}$ or if $\mu$ is small enough.

Proof. See appendix.

Typically when $\mu$ is small and $\bar{p}$ is large, the network will induce the LB content providers to trade at a positive retail price. The reason is that the network captures all the profit of the LB content (due to content competition) and that inducing monetary transactions involves little distortion when $\mu$ is small but better signals transmitted to consumers. Thus the informational value of prices is sufficient to offset the transaction cost.

When $\mu$ is large, it may happen that the optimal choice of tariff under exclusion leads to a price $p_{\ell}$ larger than the maximal willingness to pay $\bar{p}$. In this case the network chooses between free LB content and exclusion of the LB content. Without surprise, for $\mu$ very large, a free content is not viable and the analysis of the network behavior is the same as in the previous section (exclusion occurs for $\lambda>\lambda^{*}$ ).

Thus, with endogenous business model, the analysis is similar except that the LB content is excluded from the free segment but not necessarily from the market. Notice that under exclusion, welfare writes as

$$
W_{\lambda}^{p}\left(s^{p}\right)=V_{\lambda}^{p}\left(s^{p}\right)+\lambda\left(b_{h}-s^{p}\right) \theta_{h} D(0) \geq W_{\lambda}\left(b_{h}\right)
$$

Welfare is higher than with free content for two reasons. First the LB content is consumed. Second the price for content is lower. Still the network chooses excessively high prices for content. Indeed lowering the price $s$ would benefit the $\mathrm{HB}$ content providers, an effect not taken into account by the network. Hence some price-cap is optimal. Notice that for $\mu$ small, a price-cap at cost $s \leq 1$ induces prices $r=0, p_{\ell}=\left(1-b_{\ell}\right) \theta_{\ell}(1+\mu)$ and $p_{h}=0$ which are close to the socially optimal price with no transaction cost. Hence we obtain:

Proposition 11 For a given $\lambda$ and a transaction cost $\mu$ not too large, a price-cap $\sigma=1$ on the price s socially dominates laissez-faire.

Proof. Suppose that the network chooses the price-cap, then it would choose $s^{p}>1$ with no regulation. Thus a price-cap dominates as $W_{\lambda}^{p}(1)=\max _{s>b_{\ell}} W_{\lambda}^{p}(s)>W_{\lambda}^{p}\left(s^{p}\right)$. The network chooses the price-cap when

$$
S\left(\left(1-b_{\ell}\right) \theta^{e}\right)>(1-\lambda) S\left(\left(1-b_{\ell}\right) \theta_{\ell}(1+\mu)\right)+\lambda S(0)
$$


hence when $\mu$ is small.

Hence social optimality in the case of paid content with low transaction costs requires that the content providers pay for traffic but no more than the cost. It generates best retail price signal for consumers by inducing self-selection of content providers. This conclusion would be straightforward if all contents were sold at positive price, and the same logic extends to the case were some prices are optimally set to zero. ${ }^{17}$

Notice however that for a larger transaction cost $\mu$, the same issue as in the case of freecontent may arise: a price-cap at cost may induce the network to reduce excessively the price charged to content providers for traffic and raises the price charged to consumers, leading to insufficient consumption of the $H B$ contents.

However our conclusion that some positive price-cap regulation is optimal is robust.

\section{Conclusion}

This paper has investigated the impact of missing content prices for the efficient pricing of transmission network services. It has been shown that, when consumers control their consumption but are not aware of the induced effect, a direct or indirect signal should be send to them. In the standard setting with paid goods, this signal is sent through the price chosen by the content provider, but when the goods are free, this is not feasible and the network prices must substitute for the missing content price. In this context our analysis points to some interesting facts.

First, as ISPs provide unique access to consumers, even competitive ISPs will not choose fully efficient tariffs and will induce excessive exclusion of contents. This conclusion is similar to results obtained for telecommunication termination charges ${ }^{18}$, although we focus on efficiency of consumption in the presence of adverse selection.

While a zero price for content is not optimal, a price-cap on this price may improve efficiency by reducing exclusion. Then we extended our inquiry by allowing the network to propose a menu of tariff, among which each content provider must choose. By letting each content provider choosing not only its own price but also the price paid by their consumers, category pricing avoids the exclusion of the traffic intensive content and raises the volume for the less traffic intensive content.

Our analysis suggests that some regulation is optimal, although it should be light. In particular, imposing that at least one tariff proposed to content providers falls below a price-cap may reduce excessive exclusion while preserving flexibility in offers and screening

\footnotetext{
${ }^{17}$ Notice however that we assume perfect competition which is not the standard situation on Internet.

${ }^{18}$ Charges for terminating a call initiated by an other network's user toward a client of the network.
} 
possibilities.

\section{References}

[1] Akerlof, G., 1970. "Market for lemons: quality uncertainty and the market mechanism", The Quarterly Journal of Economics, Vol. 83 (3), 488-500.

[2] Armstrong, M., 2006. "Competition in Two-Sided Market", The Rand Journal of Economics, Vol. 37 (3), 668-691.

[3] Bourreau, M., Kourandi, F. and T. Valletti, 2014. "Net Neutrality with Competing Platforms", CEIS Tor Vergata Research Paper 12(3)-307.

[4] Caillaud, B., and B., Jullien, 2003. "Chicken \&Egg: competition among intermediation service providers", The Rand Journal of Economics, Vol. 34(2), 309-328.

[5] Choi, J.P., and B.-C., Kim, 2010. "Net Neutrality and Investment Incentives", The RAND Journal of Economics, Volume 41, Issue 3, pages 446-471.

[6] Choi, J.P., Jeon D.-S. and B.-C., Kim, 2013. "Asymmetric Neutrality Regulation and Innovation at the Edges: Fixed vs.Mobile Networks", mimeo, Toulouse School of Economics.

[7] Economides, N. and B., Hermalin, 2012. "The Economics of Network Neutrality", New York University Law and Economics Working Papers

[8] Economides N. and J. Tåg, 2012. "Net Neutrality on the Internet : A two-sided Market Analysis", Information Economics and Policy, vol. 24 (2012) pp. 91-104

[9] Hermalin, B. and M.L. Katz, 2004. "Sender or Receiver: who should pay to exchange an electronic message", The Rand Journal of Economics, Vol. 35(3), 423-448.

[10] Hermalin, B. and M.L. Katz, 2007. "The Economics of product-line restrictions with an application to the network neutrality debate", Information Economics and Policy, Vol. $38,215-248$.

[11] Hermalin, B. and M.L. Katz, 2009. "Information and the hold-up problem", The Rand Journal of Economics, Vol. 40, 215-248.

[12] Jullien, B., and T., Mariotti, 2006. "Auction and the informed seller problem", Games and Economics Behavior, 225-258. 
[13] Jeon, D.S., Laffont, J.J., and J. Tirole (2004). "On the Receiver-Pays Principle", RAND Journal of Economics, 35, 85-110.

[14] Krämer, J. and L. Wiewiorra, 2012. "Net neutrality and congestion sensitive content providers: Implications for content variety, broadband investment, and regulation", Information Systems Research 23(4):1303-1321.

[15] Maskin, E., 1999. "Nash Equilibrium and Welfare Optimality", Review of Economic Studies, Vol. 66, 23-38.

[16] Peitz, M. and F. Schuett, 2013. "Net neutrality and inflation of traffic", mimeo, University of Mannheim.

[17] Reggiani, C. and T. Valletti, 2012. "Net neutrality and innovation at the core and at the edges", Economic Discussion Paper EDP-1202, The University of Manchester.

[18] Rochet J.C. and J.Tirole, 2003. "Platform Conpetition in Two-Sided Markets", Journal of the European Economic Association, Vol. 1, 990-1029.

[19] Rochet J.C. and J.Tirole, 2006. "Two-sided market: A progress Report", RAND Journal of Economics, 37 (3), 645-667.

[20] Rothshild M. and J. Stiglitz (1976). "Equilibrium in Competitive Insurance Markets: An Essay on the Economics of Imperfect Information", The Quarterly Journal of Economics, Vol. 90 (4), 629-649.

[21] Samuelson, L., (1984). "Bargaining under Asymmetric Information", Econometrica, Vol. $52(4), 995-1006$.

\section{A Appendix A}

\section{Proof of proposition 2}

In each range $b \leq b_{\ell}$ and $b_{\ell}<b \leq b_{h}$, we have $r=\max \{1-s, 0\}$ which decreases with s. Moreover,

$$
\begin{aligned}
W & =M(s)[S(r \mathbb{E}(\theta \mid s))+(r \mathbb{E}(\theta \mid s)+\mathbb{E}(a \mid s)-\mathbb{E}(\theta \mid s)) D(r \mathbb{E}(\theta \mid s))] \\
& =V(s)+M(s)[\mathbb{E}(a \mid s)-s \mathbb{E}(\theta \mid s)] D(r \mathbb{E}(\theta \mid s))
\end{aligned}
$$


We have on each range

$$
\frac{\partial W}{\partial s}=M(s)\left[(r \mathbb{E}(\theta \mid s)+\mathbb{E}(a \mid s)-\mathbb{E}(\theta \mid s)) D^{\prime}(r \mathbb{E}(\theta \mid s))\right] \mathbb{E}(\theta \mid s) \frac{\partial r}{\partial s}
$$

The slope on the range is zero if $s>1$ and it is positive if $s \leq 1$ because if $s<b_{\ell}$,

$$
\begin{aligned}
r \mathbb{E}(\theta \mid s)+\mathbb{E}(a \mid s)-\mathbb{E}(\theta \mid s) & =(1-s) \theta^{e}+\lambda\left(b_{h} \theta_{h}-b_{\ell} \theta_{\ell}\right)+b_{\ell} \theta_{\ell}-\theta^{e} \\
& >-b_{\ell} \theta_{\ell}+\lambda\left(b_{h} \theta_{h}-b_{\ell} \theta_{\ell}\right)+b_{\ell} \theta_{\ell}>0
\end{aligned}
$$

while if $b_{\ell}<s \leq b_{h}$

$$
\begin{aligned}
r \mathbb{E}(\theta \mid s)+\mathbb{E}(a \mid s)-\mathbb{E}(\theta \mid s) & =\max \{1-s, 0\} \theta_{h}+b_{h} \theta_{h}-\theta_{h} \\
& =\max \left\{b_{h}-s, b_{h}-1\right\} \theta_{h}>0 .
\end{aligned}
$$

For $b_{\ell} \geq 1$, the optimum is achieved under full participation when $r=0$, thus when $1 \leq s \leq b_{\ell}$

Now suppose that $b_{\ell}<1$, then the optimum is either $b=b_{\ell}$ or exclusion with $b=b_{h}$ if it is less than 1 and $b \in\left[1, b_{h}\right]$ otherwise. In the case of exclusion the welfare is equal to the network value $V_{\lambda}\left(b_{h}\right)$ and it is linear in $\lambda$.

Consider then full participation. As $b_{\ell}<1$, welfare is

$$
W_{\lambda}\left(b_{\ell}\right)=S\left(\left(1-b_{\ell}\right) \theta^{e}\right)+\lambda\left(b_{h}-b_{\ell}\right) \theta_{h} D\left(\left(1-b_{\ell}\right) \theta^{e}\right)
$$

which is convex in $\lambda$ because $S$ is convex in prices and $D$ is convex by assumption.

Then we have $W_{0}\left(b_{\ell}\right)>W_{0}\left(b_{h}\right)=0$ and $W_{1}\left(b_{\ell}\right)<W_{1}\left(b_{h}\right)$ (because $r$ is lower at $\left.b_{h}\right)$. This implies that $W_{\lambda}\left(b_{\ell}\right)<W_{\lambda}\left(b_{h}\right)$ for $\lambda$ above a threshold $\lambda^{w}$ strictly between 0 and 1 .

\section{Proof of proposition 3}

Expected welfare for $\sigma<1$ is

$$
\begin{aligned}
W(\sigma)= & \int_{0}^{\lambda^{c}(\sigma)}\left\{S\left(\left(1-b_{\ell}\right) \theta^{e}(\lambda)\right)+\lambda\left(b_{h}-b_{\ell}\right) \theta_{h} D\left(\left(1-b_{\ell}\right) \theta^{e}(\lambda)\right)\right\} f(\lambda) d \lambda \\
& +\left\{S\left((1-\sigma) \theta_{h}\right)+\left(b_{h}-1\right) \theta_{h} D\left((1-\sigma) \theta_{h}\right)\right\} \int_{\lambda^{c}(\sigma)}^{1} \lambda f(\lambda) d \lambda
\end{aligned}
$$

leading to 


$$
\begin{aligned}
W(1)-W(\sigma) & =\int_{\lambda_{\sigma}}^{\lambda^{c}(1)}\left\{\begin{array}{c}
S\left(\left(1-b_{\ell}\right) \theta^{e}(\lambda)\right)+\lambda\left(b_{h}-b_{\ell}\right) \theta_{h} D\left(\left(1-b_{\ell}\right) \theta^{e}(\lambda)\right) \\
-\left\{S\left((1-\sigma) \theta_{h}\right)+\left(b_{h}-1\right) \theta_{h} D\left((1-\sigma) \theta_{h}\right)\right\}
\end{array}\right\} f(\lambda) d \lambda \\
& +\left\{S(0)+\left(b_{h}-1\right) \theta_{h} D(0)-S\left((1-\sigma) \theta_{h}\right)-\left(b_{h}-1\right) \theta_{h} D\left((1-\sigma) \theta_{h}\right)\right\} \int_{\lambda^{c}(1)}^{1} \lambda f(\lambda) d \lambda .
\end{aligned}
$$

The second bracket term is positive for all $\sigma<1$. The first bracket term is negative for $\sigma$ close to 1 because $\lambda^{c}(1)<\lambda_{w}$ and may be positive for smaller $\sigma$. We have

$$
\begin{aligned}
W(1)-W(\sigma) & \geq F\left(\lambda^{c}(1)\right)\left(S\left(\left(1-b_{\ell}\right) \min \left(\theta_{\ell}, \theta_{h}\right)\right)-\left\{S(0)+\left(b_{h}-1\right) \theta_{h} D(0)\right\}\right) \\
& +\left\{S(0)+\left(b_{h}-1\right) \theta_{h} D(0)-S\left((1-\sigma) \theta_{h}\right)-\left(b_{h}-1\right) \theta_{h} D\left((1-\sigma) \theta_{h}\right)\right\} \lambda^{c}(1)\left(1-F\left(\lambda^{c}(1)\right)\right)
\end{aligned}
$$

which is positive if $F\left(\lambda^{c}(1)\right)$ is small enough, implying $\sigma=1$ in this case.

For the other case, it cannot be optimal to set $\sigma=b_{\ell}$ because

$$
W^{\prime}\left(b_{\ell}\right)=\left(1-b_{\ell}\right) \theta_{h} D\left(\left(1-b_{\ell}\right) \theta_{h}\right) f\left(\lambda^{c}(\sigma)\right) \frac{d \lambda^{c}(\sigma)}{d \sigma}<0
$$

\section{Proof of proposition 4}

Note first that $\lambda^{*}$ is such

$$
\lambda^{*} \max _{R}\left[S(R)+\left(R+\left(b_{h}-1\right) \theta_{h}\right) D(R)\right]=S\left(\left(1-b_{\ell}\right) \theta^{e}\left(\lambda^{*}\right)\right)
$$

whereas $\lambda_{0}$ is such that

$$
\lambda_{0} \max _{R}\left[S(R)+\left(R+\left(b_{h}-1\right) \theta_{h}\right) D(R)\right]=S\left(\theta^{e}\left(\lambda_{0}\right)\right)+a^{e}\left(\lambda_{0}\right) D\left(\theta^{e}\left(\lambda_{0}\right)\right) .
$$

Thus $\lambda^{*}>\lambda_{0}$ if:

$$
S\left(\left(1-b_{\ell}\right) \theta^{e}\left(\lambda^{*}\right)\right)>S\left(\theta^{e}\left(\lambda^{*}\right)\right)+a^{e}\left(\lambda^{*}\right) D\left(\theta^{e}\left(\lambda^{*}\right)\right)
$$

Notice that $b_{\ell} \theta^{e}<a^{e}$ so that the comparison is ambiguous.

Suppose now that $b_{\ell}$ is close to $b_{h}$ (assuming $\theta_{h}>\theta_{\ell}$, and $b_{h}$ less than or close to 1 ). Then for all $\lambda$

$$
S\left(\theta^{e}\right)+b_{\ell} \theta^{e} D\left(\theta^{e}\right) \simeq S\left(\theta^{e}\right)+a^{e} D\left(\theta^{e}\right) \text { as } b_{\ell} \theta^{e} \simeq a^{e}
$$


But since $\theta^{e} \neq\left(1-b_{\ell}\right) \theta^{e}$,

$$
S\left(\left(1-b_{\ell}\right) \theta^{e}\right)=\max _{R}\left[S(R)+\left(R-b_{\ell} \theta^{e}-\theta^{e}\right) D(R)\right]>S\left(\theta^{e}\right)+b_{\ell} \theta^{e} D\left(\theta^{e}\right) .
$$

Hence $\lambda^{*}>\lambda_{0}$.

Consider now the case where $b_{\ell}$ is small and take $b_{\ell}=0$ as a limit case, along with $b_{h}>0$. Note then that for any $\lambda>0$ :

$$
S\left(\left(1-b_{\ell}\right) \theta^{e}\right)=S\left(\theta^{e}\right)<S\left(\theta^{e}\right)+a^{e} D\left(\theta^{e}\right) .
$$

Therefore, $\lambda_{0}>\lambda^{*}$ in this case.

At last, suppose that $b_{h}$ is large,

$$
\lambda^{*}=\frac{S\left(\left(1-b_{\ell}\right) \theta^{e}\left(\lambda^{*}\right)\right)}{S(0)+\left(b_{h}-1\right) \theta_{h} D(0)}
$$

implying that so $\lim _{b_{h} \rightarrow+\infty} \lambda^{*}=0$ and

$$
\lambda^{*} b_{h} \underset{b_{h} \rightarrow+\infty}{\rightarrow} \frac{S\left(\left(1-b_{\ell}\right) \theta_{\ell}\right)}{\theta_{h} D(0)}
$$

Remark that $a^{e}=\lambda \theta_{h} b_{h}+(1-\lambda) \theta_{\ell} b_{\ell}$ therefore $\lambda_{0}$ can be written for $b_{h}>1$ as

$$
\lambda_{0}=\frac{S\left(\theta^{e}\right)+\left(\lambda_{0} \theta_{h} b_{h}+\left(1-\lambda_{0}\right) \theta_{\ell} b_{\ell}\right) D\left(\theta^{e}\right)}{S(0)+\left(b_{h}-1\right) \theta_{h} D(0)} .
$$

which implies that $\lambda_{0}$ tends to zero and

$$
\lim _{b_{h} \rightarrow+\infty} \lambda_{0} b_{h}=\frac{S\left(\theta_{\ell}\right)+\theta_{\ell} b_{\ell} D\left(\theta_{\ell}\right)}{\theta_{h}\left(D(0)-D\left(\theta_{\ell}\right)\right)} .
$$

Thus $\lambda_{0}>\lambda^{*}$ if

$$
\frac{D(0)}{D(0)-D\left(\theta_{\ell}\right)}>\frac{S\left(\left(1-b_{\ell}\right) \theta_{\ell}\right)}{S\left(\theta_{\ell}\right)+\theta_{\ell} b_{\ell} D\left(\theta_{\ell}\right)}
$$

which hold for $\theta_{\ell}$ small but does not hold for $\theta_{\ell}$ large (close to $\bar{p}$ )

To sum up, for $b_{\ell}$ close to $b_{h}, \lambda^{*}>\lambda_{0}$ while for $b_{\ell}$ small, $\lambda_{0}>\lambda^{*}$.

\section{Proof of proposition 5}

The network compares $V_{\lambda}\left(b_{\ell}\right)$ with $V_{\lambda}(1)$. The same reasoning as for proposition 1 shows that there is a threshold $\lambda^{c}(1)$ between 0 and 1 . Then $\lambda^{c}(1)>\lambda^{*}$ for $b_{h}>1$ because 
the regulation constrains on the exclusionary price. It follows that cost orientation weakly dominates laissez-faire if $\lambda^{c}(1)<\lambda^{w}$. We then have

$$
\begin{aligned}
W_{\lambda}\left(b_{\ell}\right) & =V_{\lambda}\left(b_{\ell}\right)+\lambda\left(b_{h}-b_{\ell}\right) \theta_{h} D\left(\left(1-b_{\ell}\right) \theta^{e}\right) \\
W_{\lambda}(1) & =V_{\lambda}(1)+\lambda\left(b_{h}-1\right) \theta_{h} D(0)
\end{aligned}
$$

Thus $\lambda^{w}<\lambda^{c}(1)$ if and only if at $\lambda^{w}$, the network prefers full participation to exclusion with $s=1$ :

$$
V_{\lambda^{w}}\left(b_{\ell}\right)-V_{\lambda^{w}}(1)=\lambda \theta_{h}\left[\left(b_{h}-1\right) D(0)-\left(b_{h}-b_{\ell}\right) D\left(\left(1-b_{\ell}\right) \theta^{e}\left(\lambda^{w}\right)\right)\right]>0
$$

which gives the condition (recall that $\lambda^{w}$ depends on $b_{h}$ )

$$
b_{h}>1+\frac{\left(1-b_{\ell}\right)}{1-\frac{D\left(\left(1-b_{\ell}\right) \theta^{e}\left(\lambda^{w}\right)\right)}{D(0)}}>0
$$

which holds for $b_{h}$ large and not for $b_{h}$ close to 1 . Proof of proposition 5 The network compares $V_{\lambda}\left(b_{\ell}\right)$ with $V_{\lambda}(1)$. The same reasoning as for proposition 1 shows that there is a threshold $\lambda^{c}(1)$ between 0 and 1 . Then $\lambda^{c}(1)>\lambda^{*}$ for $b_{h}>1$ because the regulation constrains on the exclusionary price. It follows that cost orientation weakly dominates laissez-faire if $\lambda^{c}(1)<\lambda^{w}$. We then have

$$
\begin{aligned}
W_{\lambda}\left(b_{\ell}\right) & =V_{\lambda}\left(b_{\ell}\right)+\lambda\left(b_{h}-b_{\ell}\right) \theta_{h} D\left(\left(1-b_{\ell}\right) \theta^{e}\right) \\
W_{\lambda}(1) & =V_{\lambda}(1)+\lambda\left(b_{h}-1\right) \theta_{h} D(0)
\end{aligned}
$$

Thus $\lambda^{w}<\lambda^{c}(1)$ if and only if at $\lambda^{w}$, the network prefers full participation to exclusion with $s=1$ :

$$
V_{\lambda^{w}}\left(b_{\ell}\right)-V_{\lambda^{w}}(1)=\lambda \theta_{h}\left[\left(b_{h}-1\right) D(0)-\left(b_{h}-b_{\ell}\right) D\left(\left(1-b_{\ell}\right) \theta^{e}\left(\lambda^{w}\right)\right)\right]>0
$$

which gives the condition (recall that $\lambda^{w}$ depends on $b_{h}$ )

$$
b_{h}>1+\frac{\left(1-b_{\ell}\right)}{1-\frac{D\left(\left(1-b_{\ell}\right) \theta^{e}\left(\lambda^{w}\right)\right)}{D(0)}}>0
$$

which holds for $b_{h}$ large and not for $b_{h}$ close to 1 .

\section{Proof of lemma 6}

For $\lambda \leq \tilde{\lambda}$, category pricing is constrained to be equal to uniform pricing so we focus 
on the other cases. The pooling allocation is $s=b_{\ell}<1$ and $r=1-b_{\ell}$ with demand $D\left(\left(1-b_{\ell}\right) \theta^{e}\right)$. Consider the following separating allocation

$$
\begin{aligned}
s_{\ell} & =b_{\ell} ; q_{\ell}=D\left(\left(1-b_{\ell}\right) \theta_{\ell}\right) \\
s_{h} & =b_{h}\left(1-\frac{q_{\ell}}{q_{h}} \frac{\theta_{\ell}-\theta_{h}}{\theta_{\ell}}\right) ; q_{h}=D\left(\max \left\{1-s_{h}, 0\right\} \theta_{h}\right)
\end{aligned}
$$

We first claim that such an allocation exists and $s_{h}>b_{\ell}$. To see that define $\sigma(s)$ as

$$
\sigma(s)=b_{h}\left(1-\frac{q_{\ell}}{D\left\{\max (1-s, 0) \theta_{h}\right\}} \frac{\theta_{\ell}-\theta_{h}}{\theta_{\ell}}\right)
$$

This mapping is non-decreasing with $s$, with value $\sigma\left(b_{\ell}\right)>b_{\ell}$ and maximal value $\sigma(1)=$ $\sigma\left(b_{h}\right)=b_{h}\left(1-\frac{q_{\ell}}{D(0)} \frac{\theta_{\ell}-\theta_{h}}{\theta_{\ell}}\right)<b_{h}$. Hence there exists $s_{h}>b_{\ell}$ solution of $\sigma\left(s_{h}\right)=s_{h}$ which is the value in the separating allocation.

The value $V$ under pooling is (by convexity of $S$ )

$$
\begin{aligned}
S\left(\left(1-b_{\ell}\right) \theta^{e}\right) & <(1-\lambda) S\left(\left(1-b_{\ell}\right) \theta_{\ell}\right)+\lambda S\left(\left(1-b_{\ell}\right) \theta_{h}\right) \\
& <(1-\lambda) S\left(\left(1-b_{\ell}\right) \theta_{\ell}\right)+\lambda\left[S\left(\left(1-b_{\ell}\right) \theta_{h}\right)+\left(-b_{\ell}+s_{h}\right) \theta_{h} D\left(\left(1-b_{\ell}\right) \theta_{h}\right)\right] \\
& <(1-\lambda) S\left(\left(1-b_{\ell}\right) \theta_{\ell}\right)+\lambda \max _{r}\left\{S\left(r \theta_{h}\right)+\left(r+s_{h}-1\right) \theta_{h} D\left(r \theta_{h}\right)\right\} .
\end{aligned}
$$

The latter value is the value under the separating allocation. As category pricing induces a zero consumption of LB content for $\lambda \geq \bar{\lambda}$, there is exclusion of this type of content in this case.

Finally, from the definition of $\bar{\lambda}$,

$$
\frac{d \bar{\lambda}}{d a}=\frac{-1}{\left(S\left(\left(\theta_{\ell}-a\right)\right)\right)+a\left(1 / \theta_{h}-1 / \theta\right) D(0)}\left(\frac{\theta_{\ell}-\theta_{h}}{\theta_{\ell}}\right)\left(\bar{p}-\theta_{\ell}\right)<0
$$

Therefore an increase in the ad revenues decreasing the threshold above which LB content are de facto excluded.

\section{Proof of proposition 6}

From above, we only need to show that $\bar{\lambda}>\lambda^{*}$. We have

$$
S\left(\left(1-b_{\ell}\right) \theta^{e}\left(\lambda^{*}\right)\right)=\lambda^{*}\left[S(0)+\left(b_{h}-1\right) \theta_{h} D(0)\right]
$$

and

$$
\bar{\lambda}=\frac{\bar{p}-\left(1-b_{\ell}\right) \theta_{\ell}}{\bar{\lambda} \bar{p}-\left(1-b_{\ell}\right) \theta_{\ell}+\left(b_{h}-b_{\ell}\right) \theta_{h}}>\hat{\lambda}=\frac{b_{\ell} \theta_{\ell}}{b_{\ell} \theta_{\ell}+\left(b_{h}-b_{\ell}\right) \theta_{h}}
$$


But

$$
S\left(\left(1-b_{\ell}\right) \theta^{e}(\bar{\lambda})\right)<S(0)-\left(1-b_{\ell}\right) \theta^{e}(\bar{\lambda}) D(0)
$$

But direct computation shows that

$$
\begin{aligned}
& S(0)-\left(1-b_{\ell}\right) \theta^{e}(\bar{\lambda}) D(0)-\bar{\lambda}\left(S(0)+\left(b_{h}-1\right) \theta_{h} D(0)\right) \\
= & \frac{\left(b_{h}-b_{\ell}\right) \theta_{h}}{\bar{\lambda} \bar{p}-\left(1-b_{\ell}\right) \theta_{\ell}+\left(b_{h}-b_{\ell}\right) \theta_{h}}(S(0)-\bar{p} D(0))<0
\end{aligned}
$$

where the last inequality follows from $S(0)=\int_{0}^{\bar{p}} D(p) d p$. Hence

$$
S\left(\left(1-b_{\ell}\right) \theta^{e}(\bar{\lambda})\right)<\bar{\lambda}\left[S(0)+\left(b_{h}-1\right) \theta_{h} D(0)\right]
$$

implying that $\bar{\lambda}>\lambda^{*}$.

\section{Proof of proposition 7}

We focus here on the case where $\lambda<\lambda^{*}$ as it has been show in the text that category pricing is optimal for $\lambda \geq \lambda^{*}$. We need to compare social welfare with category pricing and with uniform tariff. In the first case expected social welfare is given by

$$
\begin{aligned}
W_{\lambda}^{D} & =\lambda\left[S(0)+\left(b_{h}-1\right) \theta_{h} D(0)\right]+(1-\lambda)\left[S\left(r_{\ell} \theta_{\ell}\right)+\left(r_{\ell} \theta_{\ell}+b_{\ell} \theta_{\ell}-\theta_{\ell}\right) D\left(r_{\ell} \theta_{\ell}\right)\right] \\
\text { where } r_{\ell} & =\arg \max _{r} S\left(r \theta_{\ell}\right)+\left(r \theta_{\ell}+b_{\ell} \theta_{\ell}-\theta_{\ell}-\frac{\lambda}{1-\lambda} \theta_{h}\left(b_{h}-b_{\ell}\right)\right) D\left(r \theta_{\ell}\right) .
\end{aligned}
$$

As for the case with uniform pricing, expected social welfare is given by

$$
W_{\lambda}\left(b_{\ell}\right)=S\left(\left(1-b_{\ell}\right) \theta^{e}\right)+\lambda\left(b_{h}-b_{\ell}\right) \theta_{h} D\left(\left(1-b_{\ell}\right) \theta^{e}\right)
$$

At zero we have $W_{0}^{D}=W_{0}\left(b_{\ell}\right)$. Using the first order conditions above, one can show that

$\left.\frac{\partial\left(W_{\lambda}^{D}-W_{\lambda}\left(b_{\ell}\right)\right)}{\partial \lambda}\right|_{\lambda=0}=S(0)+\left(b_{h}-1\right) \theta_{h} D(0)-\left[S\left(\left(1-b_{\ell}\right) \theta_{\ell}\right)+\left(\left(1-b_{\ell}\right) \theta_{\ell}+\left(b_{h}-1\right) \theta_{h}\right) D\left(\left(1-b_{\ell}\right) \theta_{\ell}\right)\right]$

The function $S(x)+\left(x+\left(b_{h}-1\right) \theta_{h}\right) D(x)$ is decreasing on $x \geq 0$ because $b_{h}>1$. Given that $\left(1-b_{\ell}\right) \theta_{\ell}>0$, the above slope is positive. Therefore, at least for small values of $\lambda$, category pricing welfare dominates uniform pricing. 


\section{Proof of proposition 10}

First notice that the network prefers $s=b_{h}$ to $s>b_{h}$. The reason is that this avoids paying the transaction cost. Moreover the profit of all content providers is zero in both cases, so that the network captures the total surplus.

Consider the case $s>b_{\ell}$ and $r=\max (1-s, 0)$. Let $V_{\lambda}^{p}(s)$ denote the network value:

$$
\begin{aligned}
V_{\lambda}^{p}(s) & =(1-\lambda) S\left(\left(1-b_{\ell}\right) \theta_{\ell}(1+\mu)\right)+\lambda S\left((1-s) \theta_{h}\right) \text { for } s \in\left(b_{\ell}, 1\right], \\
V_{\lambda}^{p}(s) & =(1-\lambda)\left[S\left(p_{\ell}\right)+(s-1) \theta_{\ell} D\left(p_{\ell}\right)\right]+\lambda\left[S(0)+(s-1) \theta_{h} D(0)\right] \text { for } s \in\left[1, b_{h}\right] .
\end{aligned}
$$

Then

$V_{\lambda}^{p \prime}(s)=\lambda \theta_{h} D\left((1-s) \theta_{h}\right)>0$ for $s \in\left(b_{\ell}, 1\right)$,

$V_{\lambda}^{p \prime}(s)=(1-\lambda)\left[-D\left(p_{\ell}\right) \mu \theta_{\ell}+(s-1) \theta_{\ell}^{2} D^{\prime}\left(\left(s-b_{\ell}\right) \theta_{\ell}(1+\mu)\right)\right]+\lambda\left[\theta_{h} D(0)\right]$ for $s \in\left(1, b_{h}\right)$.

Let $s^{p}(\lambda) \in \arg \max _{s>b_{\ell}} V_{\lambda}^{p}(s)$. As $V_{\lambda}^{p \prime}(s)$ increases with $\lambda$ on $s>1, s^{p}$ is non-decreasing. Moreover $V_{0+}^{p \prime}(s) \leq 0$ while $V_{1}^{p \prime}(s)>0$, implying $s^{p}(0)=1$ and $s^{p}(1)=b_{h}$. Then $s^{p}$ is preferred by the network to $b_{\ell}$ if $\lambda \geq \lambda^{*}$ because in this case $V_{\lambda}^{p}(s)>V_{\lambda}(s)$, and if $\mu$ is small because in this case $V_{\lambda}^{p}\left(b_{\ell}+\varepsilon\right)>V_{\lambda}\left(b_{\ell}\right)$. 